\title{
Remission in Rheumatoid Arthritis
}

\author{
Rania M. Shammas • Veena K. Ranganath • \\ Harold E. Paulus
}

Published online: 10 August 2010

(C) The Author(s) 2010. This article is published with open access at Springerlink.com

\begin{abstract}
With advancing therapeutic options, achieving a state of remission has become the treatment goal in rheumatoid arthritis. Agreeing on what constitutes remission and what measures should be used to assess disease activity has remained a challenge. Multiple remission criteria have been devised and modified, all with different strengths and limitations. A consensus definition of remission will need to be achieved if we are to be able to evaluate outcomes of clinical trials and establish treatment targets for practice. Remission defined as the complete absence of disease currently may not be a realistic therapeutic goal.
\end{abstract}

Keywords Rheumatoid arthritis $\cdot$ Remission

\section{Introduction}

As new disease-modifying agents and biologic therapies are added to the treatment options in rheumatoid arthritis (RA), better outcomes are expected and reported. The current

\footnotetext{
R. M. Shammas

Division of Rheumatology, University of California Los Angeles and the Veterans Association (VA)

Greater Los Angeles Health System,

Los Angeles, CA, USA

e-mail: rshammas@mednet.ucla.edu

V. K. Ranganath

Division of Rheumatology, University of California Los Angeles, Los Angeles, CA, USA

\section{H. E. Paulus $(\square)$}

Division of Rheumatology, University of California Los Angeles, Rehabilitation Building Room 32-98, 1000 Veteran Avenue, Los Angeles, CA 90024, USA

e-mail: hpaulus@mednet.ucla.edu
}

treatment goal for RA is to achieve a state of disease remission. The concept of remission at first glance seems simple. It has been defined as "an absence of articular and extra-articular inflammation and disease activity" [1]. However, no consensus exists on what constitutes clinical remission and what indices should be used to assess disease activity. Presently, no gold standard can be uniformly applied in clinical trials or practice.

In clinical practice, a subjective definition of remission is commonly applied, one in which "we can recognize it when we see it." Three clinical domains are usually considered in making this decision: signs and symptoms of inflammation, functional impairment, and structural joint damage [2]. In a 2008 study evaluating physician and patient perspectives on remission, rheumatologists were asked, "Based on your experience, is your patient currently in remission?" while patients completed questionnaires regarding their disease activity. There was a $78.6 \%$ agreement $(\kappa, 0.53)$ between patients and physicians regarding achievement of remission. However, most of these RA patients were found to have evidence of residual RA activity based on RA Disease Activity Index scores, which called into question whether subjective clinical remission is true absence of disease or a state of low disease activity [3].

One of the first descriptions of remission in RA came from Short and Bauer [4] in 1948, in which patients were classified as being in remission if they were asymptomatic and if examination of the joints was negative except for residual deformity. Since that time, various criteria have been developed to define remission using different clinical, laboratory, and radiographic measures. Clinical trials have used different definitions of remission, making it difficult to compare results between studies. To standardize the remission measure, the American College of Rheumatology (ACR) and the European League Against Rheumatism 
(EULAR) are currently collaborating to redefine remission in RA and to create a uniform definition that can be used in trials and clinical practice. They believe that a strict definition of remission is needed and should be validated against long-term outcomes [5••]. This review discusses current debates in remission, existing definitions, and their limitations.

\section{Current Remission Criteria}

Groups such as the ACR, EULAR, and the US Food and Drug Administration (FDA) have all developed different criteria to define remission (Tables 1 and 2).

\section{ACR Criteria}

One of the early attempts to define remission in RA was adopted by the American Rheumatism Association (now ACR) in 1981. Remission required fulfillment of 5 of 6 variables for at least 2 consecutive months: less than $15 \mathrm{~min}$ of morning stiffness, no fatigue, no joint pain by history, no joint tenderness, no swelling, and an erythrocyte sedimentation rate (ESR) of less than $30 \mathrm{~mm} / \mathrm{h}$ for women and less than $20 \mathrm{~mm} / \mathrm{h}$ for men [1]. Three of these six variables rely on the patient's history. It also should be noted that these criteria were established at a time when available treatment options did not provide the degree of improvement that is currently expected and possible [6].

Prevoo et al. [7] proposed modifying the ACR criteria because no specifications were provided on how to measure the variables, and because fatigue and morning stiffness are not currently recognized and accepted in a validated core set. Fatigue was removed, and having no joint pain by history was replaced by pain assessment on a Visual Analogue Scale (VAS). Remission required meeting four of the five variables of the revised criteria for 3 months [7]. One criticism regarding the ACR remission criteria is that additional factors that may contribute to joint pain (eg, structurally damaged joints) may not necessarily reflect ongoing disease activity. Moreover, it would be possible to fulfill remission criteria while still having swollen joints [6]. In addition, clinical trials do not routinely gather all data required to evaluate ACR remission criteria, and several studies have modified assessments of ACR remission criteria, excluding fatigue, stiffness, or modifying pain VAS measures [8]. Despite these limitations, the ACR remission criteria are still viewed as reliable outcome measures.

The ACR remission criteria should not be confused with the ACR response criteria. Remission is a "status" measure (ie, an assessment of disease activity at a specific point in time), whereas a "response" measure is an assessment of how disease activity changes over time. The ACR 20\%, $50 \%$, and $70 \%$ improvement criteria are response measures and were developed to evaluate a response to therapy in the context of a clinical trial, not to determine remission [9].

\section{EULAR Criteria: Disease Activity Score}

The Disease Activity Score (DAS) was proposed by EULAR in the early-1990 s. The DAS is a single-point, absolute measure of disease activity. The original DAS - the DAS44 uses four core set variables in a mathematical equation to quantify the level of disease activity. The variables include the Ritchie Tender Joint Index (a graded measurement of joint tenderness), the number of swollen joints (44 joints assessed), an acute-phase reactant (ESR or C-reactive protein [CRP]), and a general health score on a 100-mm VAS. A DAS44/ESR four-item score of less than 1.6 is considered remission. An abbreviated version, the DAS28, has been validated against the original version. A DAS28 score of less than 2.6 is considered remission [10].

The DAS28 is more time efficient, requiring the evaluation of fewer joints. However, the 28-joint count could lead to an underestimation of disease activity. Ankles and feet are commonly affected in RA but are not included in the

Table 1 ARA and modified ACR criteria for remission in rheumatoid arthritis

\begin{tabular}{|c|c|}
\hline ARA criteria ${ }^{a}$ & Modified ACR criteria ${ }^{b}$ \\
\hline 1. Morning stiffness $<15 \mathrm{~min}$ & 1. Morning stiffness $<15 \mathrm{~min}$ \\
\hline 2. No joint pain by history & 2. Pain on Visual Analogue Scale $\leq 10 \mathrm{~mm}(100 \mathrm{~mm}$ scale $)$ \\
\hline 3. No joint tenderness on examination & 3. No joint tenderness on examination \\
\hline 4. No joint or tendon sheath swelling & 4. No joint or tendon sheath swelling \\
\hline 5. ESR $<30 \mathrm{~mm} / \mathrm{h}$ (women) or $<20 \mathrm{~mm} / \mathrm{h}$ (men) & 5. ESR <30 mm/h (women) or $<20 \mathrm{~mm} / \mathrm{h}$ (men) \\
\hline 6. No fatigue & \\
\hline
\end{tabular}

${ }^{\text {a }}$ Minimum 5 of 6 over 2 mo

${ }^{\mathrm{b}}$ Minimum 4 of 5 over 3 mo

$A C R$ American College of Rheumatology, ARA American Rheumatism Association, ESR erythrocyte sedimentation rate 
Table 2 Remission criteria for rheumatoid arthritis

\begin{tabular}{|c|c|c|}
\hline Criteria & Formula used to calculate score & Required score \\
\hline DAS44 & $\mathrm{DAS} 44=0.56 * \operatorname{sqrt}(\mathrm{RAI})+0.065^{*}($ swollen 44$)+0.33^{*} \ln (\mathrm{ESR})+0.0072 * \mathrm{GH}$ & $<1.6$ \\
\hline DAS28 & DAS28 $=0.56 * \operatorname{sqrt}($ tender 28$)+0.28 * \operatorname{sqrt}($ swollen 28$)+0.70 * \ln (\mathrm{ESR})+0.014 * \mathrm{GH}$ & $<2.6$ \\
\hline SDAI & $\mathrm{SDAI}=$ swollen $28+$ tender $28+\mathrm{CRP}(\mathrm{mg} / \mathrm{dL})+$ patient $10-\mathrm{cm}$ VAS + physician $10-\mathrm{cm}$ VAS & $<3.3$ \\
\hline CDAI & $\mathrm{CDAI}=$ swollen $28+$ tender $28+$ patient $10-\mathrm{cm}$ VAS + physician $10-\mathrm{cm}$ VAS & $<2.8$ \\
\hline FDA & ACR criteria + radiographic arrest (by Larsen or Sharp method) for 6 mo after medications discontinued & - \\
\hline
\end{tabular}

$A C R$ American College of Rheumatology, CDAI Clinical Disease Activity Index, CRP C-reactive protein, DAS Disease Activity Score, ESR erythrocyte sedimentation rate, FDA US Food and Drug Administration, GH General Health score, ln logarithm, $R A I$ Ritchie Articular Index, SDAI Simplified Disease Activity Index, sqrt square root, VAS Visual Analogue Scale

assessment. Painful and/or swollen metatarsophalangeal joints have been detected in many patients classified as being in DAS28 remission [11•]. In a study comparing the DAS28 with a DAS32 that included ankles and a combined metatarsophalangeal joint count, the frequency of remission did not change between the two definitions ( $n=767,95 \%$ concordance). For those with ankle and foot involvement, abnormal acute-phase reactants or general health scores increased DAS28 scores to levels above remission [12]. However, a substantial number of RA patients can have tender and swollen joints and still be considered in DAS remission [13]. Thus, it can be argued that DAS remission reflects a measure of minimal RA disease activity rather than remission.

Another limitation of the DAS is that it relies heavily on acute-phase reactants. A subnormal ESR or CRP may artificially weigh the DAS score into remission despite objective evidence of disease activity in the joint count. Subnormal inflammatory markers may be possible given current biologic therapies that have been shown to drive down acute-phase reactants without necessarily having equivalent clinical improvement. On the flip side, ESR and CRP are nonspecific and may not accurately reflect the patient's disease activity if they are elevated for other reasons (eg, age, comorbidities). When the DAS28 is used instead of the DAS44, the acute-phase reactant becomes more heavily weighted, further amplifying changes in this variable [10].

\section{Derivatives of EULAR Criteria: Disease Activity Indices}

The Simplified Disease Activity Index (SDAI) and the Clinical Disease Activity Index (CDAI) are less complex composite indices that have been derived from the DAS. The SDAI includes five components that are simply added together for a final score: 28 tender joint count, a 28 swollen joint count, CRP, and both a patient's and a physician's global disease activity based on a $10-\mathrm{cm}$ VAS. The CDAI is exactly the same except that it excludes the CRP component and is therefore one of the few criteria that does not involve a laboratory value. Remission is defined as an SDAI score of less than 3.3 or a CDAI score of less than or equal to 2.8 [14].

An advantage of the SDAI and CDAI over the DAS is that they do not require a complex formula or a calculator and can be used more readily in clinical practice. As they are derived from the DAS, though, the disease activity indices carry similar limitations. Interestingly, RA patients who met SDAI remission criteria had less joint inflammation by power Doppler ultrasonography than patients who met DAS28 remission criteria [15]. Patients with RA in CDAI remission also have been shown to have quality-oflife scores closer to those of the healthy population when compared with patients who meet DAS28 remission criteria [16]. These studies suggest that SDAI and CDAI remission scores are stringent and may more accurately reflect a state of decreased disease activity than DAS28 remission scores.

When SDAI and CDAI were compared with the DAS28 in a clinical practice setting, significantly fewer RA patients met CDAI/SDAI remission than DAS28 remission [17]. This highlights the point that there are different remission rates depending on the definition used. In a large, multinational, cross-sectional cohort of 5848 RA patients in 24 countries, $8.6 \%$ met ACR criteria for remission, $13.8 \%$ met CDAI remission criteria, and $19.6 \%$ achieved DAS28 remission-numbers that are consistent with the ACR criteria being the most difficult to fulfill and the DAS28 being more inclusive [18••]. Therefore, clinical trials must be interpreted carefully in light of the remission definitions being used.

\section{FDA Criteria}

The FDA criteria define remission as meeting the above ACR remission criteria plus radiographic arrest (defined by Larsen or Sharp scores) for 6 months after all antirheumatic therapy has been discontinued. This is the only definition that includes radiographic changes as well as a medicationfree state. These is the most stringent of all the criteria, and no therapy to date has been able to fulfill the requirements (Fig. 1) [19]. 


\section{Stringency of criteria}

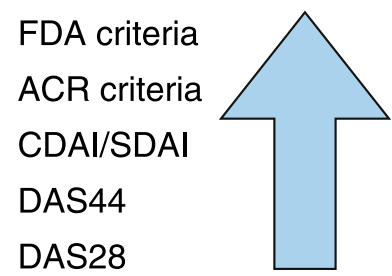

Fig. 1 Stringency of remission criteria for rheumatoid arthritis. US Food and Drug Administration (FDA) criteria are the most difficult to fulfill, whereas Disease Activity Score (DAS) 28 criteria are the most inclusive. ACR-American College of Rheumatology; CDAIClinical Disease Activity Index; SDAI-Simplified Disease Activity Index

\section{Medication-Free Remission}

The concept that remission in RA should require a diseasefree state after the discontinuation of medications is debatable. At present, the cause for the immune dysregulation that leads to RA is unknown. Current treatment aims to control the disease, not cure it. Thus, is it theoretically possible to achieve remission while being off treatment if the underlying immune pathology is still present? [20]. It is also known, however, that a wide spectrum of possible disease outcomes exists for patients with RA, ranging from mild to rapidly destructive and debilitating disease. Drugfree remission has been previously reported in a small proportion of RA patients. Within this subset of patients, it is unclear whether current therapies are inducing a greater rate of drug-free remission or if the observed percentages represent the natural history of RA subtypes. The few studies addressing drug-free remission vary in how remission is defined, making them difficult to compare [21].

Tippana-Kinnunen et al. [22••] observed 87 patients with early RA for 15 years treated by the "sawtooth strategy." Disease-modifying antirheumatic drugs (DMARDs) were discontinued in $29 \%$ of patients who met the modified ACR criteria of remission (excluding fatigue) or who had an undefined symptom-free period. However, 11 of the patients off medications (45\%) had disease flares and had to be restarted on DMARDs. The mean DMARD-free period was 50 months overall (range, 3-137). None of the patients who had to restart DMARD therapy were in remission at the end of 15 years, suggesting that it may be more difficult to control RA after medications have been stopped and then restarted. The authors concluded that most patients with long-standing RA require continuous DMARD treatment, and if the treatment is discontinued, patients should be followed up closely to monitor for flares $[22 \bullet \bullet]$.

A 4-year follow-up to the BeSt (Dutch acronym for Behandel-Strategieen treatment strategies) study looked at drug-free remission in 508 patients with recent-onset, active RA. Four different treatment strategies were adjusted every 3 months to achieve a DAS of less than 2.4. From year 2 onward, if the patient had a DAS less than 1.6 for at least 6 consecutive months, all antirheumatic treatment was discontinued. Between months 24 and 48, drug-free remission was achieved in $20 \%(n=102)$. By the end of 4 years, $13 \%$ $(n=67)$ were still in drug-free remission, with the other patients requiring reinitiation of treatment. The mean duration of drug-free remission was 9 to 11 months. Treatment strategy was not associated with drug-free remission. Patients with milder RA at baseline, the absence of autoantibodies, male gender, and shorter symptom duration were associated with drug-free remission at year 4 [23].

Another study of the Leiden Early Arthritis Clinic (EAC) and the British Early Rheumatoid Arthritis Study (ERAS) also looked at the prevalence and predictive factors related to DMARD-free remission. Remission was defined as having no swollen joints, being off DMARDs, and classified as being in remission by a rheumatologist for a total duration of 1 year. Sustained DMARD-free remission was achieved in $15 \%$ of the EAC group (68 of 454 patients) and $9.4 \%$ of the ERAS group ( 84 of 895 patients). Factors associated with remission included acute onset, short symptom duration, nonsmokers, little radiographic damage at baseline, absence of rheumatoid factor, and absence of HLA shared epitope alleles [24•].

These studies suggest that a small proportion of earlyRA patients $(9 \%-15 \%)$ will be able to achieve medicationfree remission for a sustained period of time and that certain factors may help predict in which RA patients this would be possible. However, up to $45 \%$ will require a reinitiation of therapy, and RA treatment may be more difficult when medications are restarted. These studies varied in how remission was defined, making it difficult to determine how to apply medication-free remission to clinical practice. There are no clear guidelines as to if and when medications should be stopped in RA.

\section{Imaging in Remission}

The purpose of having remission as a goal in RA treatment is to prevent joint damage and disability. Active inflammation in RA is generally correlated with progressive joint destruction. However, it has been shown that patients in clinical remission by ACR criteria [25] and by DAS28 criteria [26] can develop erosions and joint damage on radiographs of hands and feet when observed 2 to 5 years later. The progression of joint damage despite clinical remission suggests that traditional assessments of disease activity may not be sensitive enough, thereby leaving levels 
of RA activity undetected [27]. This has led to the suggestion that radiographs, ultrasound, or MRI should be included in remission criteria to better assess disease activity. At present, the FDA definition of remission is the only criterion that requires the absence of radiographic progression.

In a study using data from the PREMIER (A Multicenter, Randomized, Double-Blind Clinical Trial of Combination Therapy With Adalimumab Plus Methotrexate Versus Methotrexate Alone or Adalimumab Alone in Patients With Early, Aggressive Rheumatoid Arthritis Who Had Not Had Previous Methotrexate Treatment) trial, 794 patients with early RA on three different treatment strategies were monitored for radiographic progression. Remission as defined by CDAI, SDAI, or DAS28 was achieved in $15 \%$ of patients $(n=119)$ by the second year of the trial. Radiographic progression was significantly different between patients with 3 , 6, or 9 additional months of remission $(P<0.05)$ regardless of the type of treatment received. The shorter the duration of remission, the more likely radiographic progression was found. It was concluded that this was likely a carryover effect of past periods of inflammation and that this latency should be considered when evaluating radiographic outcomes in clinical trials and remission [28].

MRI and ultrasound are now being used more commonly to assess RA disease activity. Patients who have met the modified ACR criteria and DAS28 criteria for remission have ongoing synovitis by ultrasound [29]. Brown et al. [27] studied ultrasound and MRI images of the hands of 107 RA patients who were considered to be in remission by their rheumatologist, the DAS28, or the modified ACR criteria. Regardless of which definition of remission was used, MRI showed that $96 \%$ of patients had synovitis and $46 \%$ had bone marrow edema, while ultrasound showed that $73 \%$ had synovial hypertrophy and $43 \%$ had increased power Doppler signal. No significant difference was noted in the number of patients with inflammation between those who met DAS28 or ACR remission criteria. These findings suggest that DAS28 and ACR criteria may not measure RA remission, in that "true remission" should show the absence of synovitis on imaging studies [27].

In a follow-up longitudinal study, 102 of the RA patients thought to be in remission underwent routine radiographs, ultrasound, and MRI at 12 months. Despite apparent clinical remission, $19 \%$ of patients ( $n=17$ [of 90 analyzed]) had continued deterioration in radiographic joint damage, although 11 satisfied ACR and DAS28 remission criteria. Inflammatory scores on ultrasound, especially power Doppler signals, and MRI synovitis scores at baseline were significantly associated with development of radiographic damage over 1 year. This study documents the association of subclinical inflammation with progressive structural damage in RA patients in clinical remission. It also supports the use of sensitive imaging techniques to more accurately evaluate disease status and define the remission state $[30 \bullet \cdot$.

Subclinical inflammation detected by ultrasound and MRI may predict which patients considered to be in remission are likely to experience disease progression. In another study of RA patients in remission by DAS less than 1.6, having a positive power Doppler signal on ultrasound was the main predictor of relapse within 6 months [31•]. Imaging could be valuable in therapeutic decisions, helping clinicians decide whether patients in clinical remission should continue, stop, or restart antirheumatic medications. Currently, no guidelines exist on how to incorporate imaging in clinical practice and trials.

\section{Spontaneous Remission}

Spontaneous remission is not uncommon in patients who present with very early arthritis, some of whom may meet criteria for RA over less than a few months. Spontaneous remission is thought of as a "natural remission," in which disease activity essentially disappears, and medications are no longer required. Spontaneous remission may be seen in $13 \%$ to $55 \%$ of individuals presenting with undifferentiated arthritis, probably as a result of different underlying etiologies, such as a transient viral infection [32]. About one third of patients with undifferentiated arthritis go on to develop RA. In a study of three cohorts of recent-onset undifferentiated arthritis from the United Kingdom, Germany, and the Netherlands, the severity of morning stiffness and presence of autoantibodies were the strongest predictors of progression to RA [33]. Spontaneous remission can be seen not only in early undifferentiated arthritis but in RA pregnancy and juvenile idiopathic arthritis (JIA) as well.

\section{Pregnancy}

Pregnancy has a beneficial effect on the signs and symptoms of RA to the point at which patients can appear to have spontaneous remission of disease activity. Prospective studies have shown improvement in joint involvement in RA in two thirds to three quarters of pregnancies [34]. Improvement occurs for $50 \%$ to $76 \%$ of patients by the end of the first trimester and is usually sustained throughout pregnancy. However, within 3 months of delivery, relapse is observed in $90 \%$ of patients [35]. Most pregnant women with RA have an uneventful course with no significant complications. Except for slight intrauterine growth restriction in patients with active disease (3.36 vs $3.52 \mathrm{~kg}$ birth weight in matched controls; $P=0.01$ ), the outcome of pregnancy is favorable in patients with RA [36]. 
In a nationwide prospective study, 84 pregnant RA patients were observed during each trimester and postpartum. DAS28 scores decreased significantly $(P=0.035)$ during pregnancy and increased postpartum. In patients with DAS greater than $3.2(n=52)$, about $48 \%$ had moderate improvement based on EULAR-defined response criteria. In patients with DAS greater than 2.6 and less than $3.2(n=32)$, disease activity was stable during pregnancy. Before conception, $10 \%$ of patients were in DAS28 remission (DAS28<2.6). The number of patients in remission increased during the first trimester to $17 \%$, and $27 \%$ achieved DAS28 remission by the third trimester. However, only $18 \%$ were in remission by the 12th week postpartum. Thirty-nine percent of patients had a moderate flare postpartum. Less medication was used during pregnancy compared with before conception and postpartum. The authors showed that RA disease activity can improve during pregnancy but that not as many patients achieved disease remission as previously thought $[37 \bullet \bullet]$.

\section{Juvenile Inflammatory Arthritis}

Spontaneous remission also has been reported in JIA (a heterogeneous group of childhood diseases that involve chronic idiopathic inflammation of one or more joints). JIA has three general subgroups: oligoarthritis, polyarthritis, and systemic disease. Frequencies of remission range from $29 \%$ to $78 \%$ depending on the subtype of disease, the definition of remission, and the study being reviewed [38].

The patterns of clinical remission within categories of JIA were studied in 437 JIA patients for at least 4 years. Patients were evaluated for episodes of inactive disease, clinical remission on medication, and clinical remission off medication. A total of $89 \%$ of patients ( $n=391$ ) experienced episodes of inactive disease with a median length of 12.7 months. Those with oligoarticular JIA $(n=258)$ spent $60 \%$ of their time in a state of inactive disease, whereas those with polyarticular or systemic JIA spent on average $30 \%$ to $36 \%$ of their time in a state of inactive disease. Twenty-six percent of patients $(n=228)$ had clinical remission off medication, with $68 \%$ of this group having oligoarticular JIA. Thirty-six percent of these episodes of remission off medication persisted for 2 years, but only $6 \%$ persisted for 5 years. This study shows that spontaneous remission in JIA is not as common and sustainable as previously reported and more likely to occur in the oligoarticular subtype [39].

\section{Conclusions}

RA is a complex inflammatory disorder with a wide spectrum of manifestations and outcomes ranging from mild, limited disease to severe, debilitating disease. We may be dealing with distinct diseases leading to different probabilities of remission.
The exact etiology of RA is unknown, but further insights are being made continuously. New disease-modifying and biologic therapies are being introduced to suppress RA-associated inflammation. With more effective treatment options, the goal of treatment has become to achieve "remission." Defining remission, however, has remained a challenge. With varying definitions of what constitutes remission, it is difficult to compare results of current drug trials and to know how to apply these results to clinical practice.

The current definitions of remission have major limitations. A patient can have tender and swollen joints and still meet most criteria for remission. Furthermore, it also has been shown that subclinical inflammation may be present on imaging despite the absence of clinical findings of disease activity, which could explain continuing joint damage despite a remission state. This continued progression of disease suggests that RA patients are not achieving true remission. If remission is defined as the complete absence of disease activity, these findings question whether remission is currently an achievable target. A more realistic treatment expectation in clinical practice may be to achieve minimal disease activity rather than complete absence of disease. Telling patients with RA that they are in remission may lead to periods of undertreatment and give patients (and physicians) a false sense of security, as patients are still at risk of developing deformities. With improved therapeutic management and medication development, perhaps remission can be a realistic goal in the future.

Many questions remain unanswered. Should remission be defined as the complete absence of disease, including signs, symptoms, inflammatory markers, and imaging? Should it apply to a moment in time or require a defined duration or permanent cure? Should remission permit treatment or require a drug-free state? As previously mentioned, a combined ACR and EULAR committee is attempting to redefine remission in RA and to reach a consensus as to how to apply the definition to clinical trials and practice. Many of these same questions were proposed and discussed. The committee concluded that the new definition should be strict, based on no or very low disease activity, and that remission should be defined independent of treatment, time course, and long-term outcomes such as radiographic damage. Variables deemed to be most important were tender joint count (with more joints being included), swollen joint count, and acute-phase reactants. A research agenda and subsequent meetings were planned to further investigate how to define remission $[5 \bullet \cdot]$.

At the present time, when planning or evaluating RA therapeutic outcomes, it is important to carefully assess the precise definition of what is considered to be remission. A consensus definition of remission will help us evaluate outcomes of clinical trials and to establish treatment targets for practice. 
Acknowledgments Dr. Ranganath is supported by ACR Research and Education Foundation Career Development Bridge funding awards and has received grant funding from the National Institutes of Health.

Dr. Paulus is supported by an ACR Research and Education Foundation Within Our Reach grant.

Disclosure Dr. Ranganath has received gifts/grants from UCB, Roche, Amgen, and CycLex.

Dr. Paulus has served as a consultant for Amgen and has received grant support from Amgen and Abbott Laboratories. No other potential conflicts of interest relevant to this article were reported.

Open Access This article is distributed under the terms of the Creative Commons Attribution Noncommercial License which permits any noncommercial use, distribution, and reproduction in any medium, provided the original author(s) and source are credited.

\section{References}

Papers of particular interest, published recently have been highlighted as:

- Of importance,

-. Of major importance

1. Pinals RS, Masi AT, Larsen RA: Preliminary criteria for clinical remission in rheumatoid arthritis. Arthritis Rheum 1981, 24:13081315.

2. Paulus HE: Defining remission in rheumatoid arthritis: what is it? Does it matter? J Rheumatol 2004, 31:1-4.

3. Wolfe F, Boers M, Felson D, et al.: Remission in rheumatoid arthritis: physician and patient perspectives. J Rheumatol 2009, 36:930-933.

4. Short CL, Bauer W: The course of rheumatoid arthritis in patients receiving simple medical and orthopedic measures. N Engl J Med 1948, 238:142-148.

5. •• Van Tuyl LHD, Vlad SC, Felson DT, et al.: Defining remission in rheumatoid arthritis: results of an initial American College of Rheumatology/European League Against Rheumatism consensus conference. Arthritis Rheum 2009, 61:704-710. This article describes the ACR and EULAR collaboration to define remission in rheumatoid arthritis. It outlines the current questions regarding remission and the research agenda set out to reach a consensus definition, concluding that a strict definition needs to be used.

6. Sesin CA, Bingham CO: Remission in rheumatoid arthritis: wishful thinking or clinical reality? Semin Arthritis Rheum 2005, 35:185-196.

7. Prevoo ML, van Gestl AM, vant Hof MA, et al.: Remission in a prospective study of patients with rheumatoid arthritis. American Rheumatism Association preliminary remission criteria in relation to the Disease Activity Score. Br J Rheumatol 1996, 35:11011105.

8. Ma MHY, Scott IC, Kingsley GH, Scott DL: Remission in early rheumatoid arthritis. J Rheumatol 2010 Jun 1 (Epub ahead of print).

9. Ranganath VK, Khanna D, Paulus HE: ACR remission criteria and response criteria. Clin Exp Rheumatol 2006, 24(Suppl 43): S14-S21.

10. OmettoF,BotsiosC,RaffeinerB,etal.:Methodsusedtoassessremissionand low disease activity in rheumatoid arthritis. Autoimmun Rev 2009, 9:161164.
11. • Van der Leeden M, Steultjens MP, van Schaardenburg D, Dekker J: Forefoot disease activity in rheumatoid arthritis patients in remission: results of a cohort study. Arthritis Res Ther 2010, 12: R3. This study highlights one of the limitations of the DAS28 criteria, showing that there can be significant forefoot disease activity in patients who meet DAS28 remission criteria.

12. Kapral T, Dernoschnig F, Machold KP, et al.: Remission by composite scores in rheumatoid arthritis: are ankles and feet important? Arthritis Res Ther 2007, 9:R72.

13. Makinen H, Kautiainen H, Hannonen P, Sokka T: Is DAS28 an appropriate tool to assess remission in rheumatoid arthritis? Ann Rheum Dis 2005, 64:1410-1413.

14. Aletaha D, Smolen J: The Simplified Disease Activity Index (SDAI) and the Clinical Disease Activity Index (CDAI): a review of their usefulness and validity in rheumatoid arthritis. Clin Exp Rheumatol 2005, 23(Suppl 39):100-108.

15. Balsa A, de Miguel E, Castillo C, et al.: Superiority of SDAI over DAS-28 in assessment of remission in rheumatoid arthritis patients using power Doppler ultrasonography as a gold standard. Rheumatology 2010, 49:683-690.

16. Linde L, Sorensen J, Ostergaard M, et al.: Does clinical remission lead to normalization of EQ-5D in patients with rheumatoid arthritis and is selection of remission criteria important? J Rheumatol 2010, 37:285-290.

17. Gulfe A, Aletaha D, Saxne T, Geborek P: Disease activity level, remission and response in established rheumatoid arthritis: performance of various criteria sets in an observational cohort, treated with anti-TNF agents. BMC Musculoskelet Disord 2009, 10:41-49.

18. •- Sokka T, Hetland ML, Makinen H, et al.: Remission and rheumatoid arthritis, data on patients receiving usual care in ? twentyour $<$ ?thyc $=$ countries. Arthritis Rheum 2008, 58:26422651. This study compared the different definitions of remission in a cohort of 5848 RA patients across 24 countries. It illustrates that remission rates vary depending on the definition being used, with the DAS28 being the most inclusive and the ACR criteria the most strict.

19. Makinen H, Hannonen P, Sokka T: Definitions of remission for rheumatoid arthritis and review of selected clinical cohorts and randomized clinical trials for the rate of remission. Clin Exp Rheumatol 2006, 24(Suppl 43):22-28.

20. Valesini G, Di Franco M, Spinelli FR, Scrivo R: Induction of remission in rheumatoid arthritis. Rheumatol Int 2008, 29:131139.

21. Yvonne PM, Goekoop-Ruiterman YPM, Huizinga TW: Can we achieve true drug-free remission in patients with RA? Nat Rev Rheumatol 2010, 6:68-70.

22. •- Tippana-Kinnunen T, Paimela T, Kautanian H, et al.: Can disease modifying anti-rheumatic drugs be discontinued in longstanding rheumatoid arthritis? A 15 year follow-up. Scand J Rheumatol 2010, 39:12-18. Of the drug-free remission papers, the follow-up period of 15 years used here is the longest. DMARDS were discontinued in RA patients meeting ACR criteria, $45 \%$ of whom had to be restarted on treatment due to disease flares. None of the patients who had to restart DMARDs were in remission by the end of 15 years.

23. Van der Kooij SM, Goekoop-Ruiterman YPM, de Vries-Bouwstra $\mathrm{JK}$, et al.: Drug-free remission, functioning and radiographic damage after 4 years of response-driven treatment in patients with recent-onset rheumatoid arthritis. Ann Rheum Dis 2009, 68:914921.

24. • Van der Woude D, Young A, Jayakumar K, et al.: Prevalence of and predictive factors for sustained disease-modifying antirheumatic drug-free remission in rheumatoid arthritis, results from two large early arthritis cohorts. Arthritis Rheum 2009, 60:2262-2271. This paper demonstrated that acute onset of symptoms, short 
symptom duration, nonsmokers, little radiographic damage at baseline, and absence of rheumatoid factor are associated with higher rates of DMARD-free remission.

25. Molenaar ET, Voskuyl AE, Dinant HJ, et al.: Progression of radiologic damage in patients with rheumatoid arthritis in clinical remission. Arthritis Rheum 2004, 50:36-42.

26. Cohen G, Gossec L, Dougados M, et al.: Radiologic damage in patients with rheumatoid arthritis in sustained remission. Ann Rheum Dis 2007, 66:358-363.

27. Brown AK, Quinn MA, Karim Z, et al.: Presence of significant synovitis in rheumatoid arthritis patients with disease-modifying anti-rheumatic drug-induced clinical remission, evidence from an imaging study may explain structural progression. Arthritis Rheum 2006, 54:3761-3773.

28. Aletaha D, Funovits J, Breedveld FC, et al.: Rheumatoid arthritis joint progression in sustained remission is determined by disease activity levels preceding the period of radiographic assessment. Arthritis Rheum 2009, 60:1242-1249.

29. Ozgocmen S, Ozdemir H, Kiris A, et al.: Clinical evaluation and power Doppler sonography in rheumatoid arthritis: evidence for ongoing synovial inflammation in clinical remission. South Med J 2008, 101:240-245.

30. • Brown AK, Conaghan PG, Karim Z, et al.: An explanation of the apparent dissociation between clinical remission and continued structural deterioration in rheumatoid arthritis. Arthritis Rheum 2008, 58:2958-2967. This is an important paper showing that RA patients in remission by ACR and DAS criteria can continue to have subclinical inflammation by ultrasound and MRI. Inflammatory scores on ultrasound, especially power Doppler signals, and MRI synovitis scores at baseline were significantly associated with development of radiographic damage over 1 year, offering an explanation as to why RA patients in remission have continued structural damage over time.

31. - Scire CA, Montecucco C, Codullo V, et al.: Ultrasonographic evaluation of joint involvement in early rheumatoid arthritis in clinical remission: power Doppler signal predicts short-term relapse. Rheumatology 2009, 48:1092-1097. This study illustrates that subclinical inflammation in patients who are thought to be in remission may be a tool to help predict short-term relapse of disease activity.

32. Pincus T, Kavanaugh A, Smolen AJ: Complexities in defining remission in rheumatic disease. Clin Exp Rheumatol 2006, 24 (Suppl 43):S1-S6.

33. Van der Helm-van Mil AHM, Detert J, le Cessie S, et al.: Validation of a prediction rule for disease outcome in patients with recent-onset undifferentiated arthritis. Moving toward individualized treatment decision-making. Arthritis Rheum 2008, 58:2241-2247.

34. Gromnica-Ihle E, Østensen M: Pregnancy in patients with rheumatoid arthritis and inflammatory spondyloarthropathies. Z Rheumatol 2006, 65:209-212, 214-216.

35. Nelson JL, Østensen M: Pregnancy and rheumatoid arthritis. Rheum Dis Clin North Am 1997, 23:195-212.

36. Bowden AP, Barrett JH, Fallow W, Silman J: Women with inflammatory polyarthritis have babies of lower birth weight. J Rheumatol 2001, 28:355-359.

37. •- De Man YA, Dolhain RJ, van de Geijn FE, et al.: Disease activity of rheumatoid arthritis during pregnancy: results from a nationwide prospective study. Arthritis Rheum 2008, 59:12411248. This paper looks at the disease activity of $R A$ patients during and after pregnancy. It demonstrates the increased rate of remission as defined by DAS28 during pregnancy and the increase flare rate postpartum. It also shows that $R A$ remission in pregnancy is not achieved as frequently as previously reported.

38. Lurati A, Salmaso A, Gerloni V, et al.: Accuracy of Wallace criteria for clinical remission in juvenile idiopathic arthritis: a cohort study of 761 consecutive cases. J Rheumatol 2009, 36:1532-1535.

39. Wallace CA, Huang B, Bandeira M, et al.: Patterns of clinical remission in select categories of juvenile idiopathic arthritis. Arthritis Rheum 2005, 52:3554-3562. 